\title{
The linear-viscoelastic behaviour of a dispersion of transversely rigid spherical capsules
}

\author{
R. A. de Bruijn and J. Mellema \\ Rheology Group, Department of Applied Physics, Twente University of Technology, Enschede (The Netherlands)
}

\begin{abstract}
A rheological model has been derived for the linear-viscoelastic behaviour of a dispersion of transversely rigid spherical capsules. The model incorporates finite thickness of the elastic shell of the capsules, anisotropy of the mechanical properties of the interface and finite volume fraction. The dynamic viscosity of the dispersion is calculated. The influence of the microstructural parameters is considered and the results are compared with those of other models. The model shows that finite thickness of the shell can strongly influence the relaxation times.
\end{abstract}

Key words: Capsule, transversely rigid shell, dispersion, complex viscosity, linear viscoelasticity

\begin{tabular}{|c|c|c|c|}
\hline Noto & & $t$ & - time \\
\hline$a$ & - particle radius & $T_{r r}, T_{\phi r}$ & - components of $\boldsymbol{T}$ \\
\hline$A$ & $-6 \times 6$ matrix & $\boldsymbol{u}$ & - velocity vector \\
\hline$b$ & - radius of the cell & $u_{r}, u_{\phi}$ & - spherical components of $\boldsymbol{u}$ \\
\hline $\boldsymbol{B}$ & $-6 \times 6$ matrix & $\boldsymbol{u}^{0}$ & - velocity vector at the sample boundary \\
\hline C & $-6 \times 1$ matrix & $V$ & $-\left(=\eta^{(i)} / \eta^{(e)}\right)$ viscosity ratio \\
\hline$D$ & - rate of strain tensor & $V_{c}$ & - cell volume \\
\hline$e$ & - unit vector & $\partial V_{c}$ & - cell surface \\
\hline$E^{\cdot}$ & - Young's modulus & $V_{p}$ & - particle volume \\
\hline$f$ & - correction factor & $\partial V_{p}^{p}$ & - particle surface \\
\hline $\boldsymbol{F}_{\lambda}$ & - force vector & $V_{s}^{p}$ & - sample volume \\
\hline$g_{\lambda}$ & - scalar quantity defined in eq. (20) & $\partial V_{s}$ & - sample surface \\
\hline$G$ & - constant proportional to the applied rate of strain & $X_{1}, \ldots X_{6}$ & - functions of $R, L, V$ and $v$ \\
\hline$h$ & - shell thickness & $\boldsymbol{Y}$ & $-6 \times 1$ matrix \\
\hline$k$ & - particle index & $Y_{1}, \ldots Y_{6}$ & - components of $Y$ \\
\hline$L$ & $-\left(=h / 2 a_{m}\right)$ relative shell thickness & $Z$ & $-\left(=2 \omega \eta^{(e)} a_{m} /(E h)\right)$ \\
\hline$m_{\lambda}$ & - resultant surface moment per unit surface & $\alpha, \beta$ & - curvilinear surface coordinates \\
\hline$M_{\lambda}$ & - resultant bending moment per unit length & $\gamma_{\lambda \mu}$ & - strain component \\
\hline$M_{\lambda \mu}$ & - resultant twisting moment per unit length & $\varepsilon_{\lambda}$ & - strain component \\
\hline$n$ & - normal vector & $\zeta$ & - distance along the normal to the middle surface \\
\hline$N_{\lambda}$ & - resultant normal force per unit length & $\eta$ & - viscosity \\
\hline$N_{\lambda \mu}$ & - resultant shear force per unit length & $\eta^{*}$ & $-\left(=\eta^{\prime}-i \eta^{\prime \prime}\right)$ complex viscosity \\
\hline$p$ & - pressure & $\eta_{\mathrm{spec}}^{*}$ & $-\left(=\left(\eta^{*}-\eta^{(e)}\right) / \eta^{(e)}=\eta_{\mathrm{spec}}^{\prime}-i \eta_{\mathrm{spec}}^{\prime \prime}\right)$ specific com- \\
\hline$p_{0}$ & - equilibrium pressure & & plex viscosity \\
\hline$q_{\lambda}$ & - resultant loading force per unit surface & $\theta$ & - spherical coordinate \\
\hline$Q_{\lambda}$ & - resultant shear force per unit length & $\theta_{\lambda}$ & - strain component \\
\hline$r$ & - spherical coordinate & $x^{n}$ & - surface dilatational modulus \\
\hline$r$ & - position vector & $K_{\lambda}$ & - strain component \\
\hline$R$ & $-\left(=b / a_{m}\right)$ relative cell radius & $\mu$ & - surface shear modulus \\
\hline$R_{\lambda}$ & - radius of curvature & $v$ & - Poisson ratio \\
\hline$s$ & - displacement vector & $\sigma_{\lambda}$ & - normal force per unit surface \\
\hline$s_{z}$ & - component of $s$ & $\tau_{\lambda \mu}$ & - shear force per unit surface \\
\hline$S$ & - area & $\phi$ & - spherical coordinate \\
\hline
\end{tabular}


$\Phi$ - volume concentration

$\omega \quad-$ angular frequency

Subscripts

ext - including shell volume

int - excluding shell volume

$l \quad-$ longest

$m \quad-$ middle surface

$s \quad-$ shortest

$\alpha, \beta, \zeta \quad-$ component in $\alpha, \beta, \zeta$ direction

\section{Superscripts}

e $\quad-$ external fluid

$i \quad-$ internal fluid

$\zeta \quad-$ quantity at a distance $\zeta$ from the middle surface

\section{Introduction}

That part of the rheology of emulsions which focusses on the influence of the mechanical properties of the almost spherical interface between dispersed and continuous phase has attracted increasing interest over the last few years. A few reasonably well defined model systems are available to study interfaces experimentally. Dynamic shear experiments have been applied for the investigation of ionic and nonionic (micro-) emulsions $[1,2]$. In particular non-linear properties of capsule dispersions with cross-linked polymeric interfaces [3] have been studied. Potentially spherical vesicles would seem a suitable subject for further study.

On the theoretical side several models [4-9] are available. A survey, arranged with aid of the parame- ters volume fraction $\phi$ and relative shell thickness $L$, of these models as far as the complex viscosity is concerned is given in table 1 .

The subject of the present work was to develop a theoretical model for a capsule dispersion that incorporates finite thickness, anisotropy of the mechanical properties of the interface and finite volume fractions and which would allow us to interpret the results of dynamic shear measurements for instance of vesicles.

The considered capsules consist of a Newtonian fluid with viscosity $\eta^{(i)}$ enclosed in a spherical transversely rigid shell of thickness $h$. A capsule is surrounded by a Newtonian fluid with viscosity $\eta^{(e)}$ (see figure 1). The shell is infinitely stiff in a direction perpendicular to the surface and mechanically isotropic in a tangential direction. The mechanical properties in the shell are characterized by the Young's modulus $E$ and the Poisson ratio $v$. Transversely rigid shells simplify the relationship between forces and deformations in the hydrodynamic description of the system. It is assumed

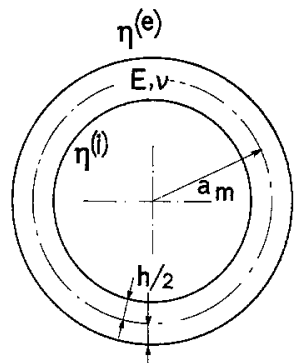

Fig. 1. Schematic picture of the capsule

Table 1. Survey of theoretical models

\begin{tabular}{|c|c|c|c|}
\hline $\begin{array}{l}\text { volume } \\
\text { fraction }\end{array}$ & $L=0 *$ ) & $L \leqq 0.05$ & $L$ arbitrary \\
\hline$\Phi$ finite & $\begin{array}{l}\text { Oosterbroek [7] } \\
-\omega \geqq 0\end{array}$ & $\begin{array}{l}\text { Present work } \\
-\omega \geqq 0 \\
- \text { transversely } \\
\quad \text { rigid }\end{array}$ & \\
\hline$\Phi \rightarrow 0$ & $\begin{array}{l}\text { Oldroyd [4] } \\
-\omega \geqq 0 \\
\text { Barthès-Biesel } \\
\text { and Chhim [8] } \\
-\omega \rightarrow 0 \\
- \text { incompressible } \\
- \text { isotropic }\end{array}$ & & $\begin{array}{l}\text { Sakanishi and Takano }[5] \\
\text { Brunn }[6] \\
-\omega \geqq 0 \\
- \text { incompressible } \\
- \text { isotropic } \\
\text { Takano and Sakanishi }[9] \\
-\omega \geqq 0 \\
- \text { incompressible } \\
- \text { anisotropic }\end{array}$ \\
\hline
\end{tabular}

*) With $L=0$ the limit described in section 5.2 is meant. 
to be applicable up to $L=h /\left(2 a_{m}\right)=0.05$ [10], where $a_{m}$ is the radius of the so-called middle surface of the shell. To be able to account for hydrodynamic interactions a cell model has been used. At the cell surface a harmonically oscillating pure straining flow is applied. The flow is so weak that the capsules remain almost spherical. For the formulation of the boundary conditions at the shell the occurring stresses and strains for small ellipsoidal deformations are considered. The complete set of six boundary conditions (at the cell surface and the shell) makes it possible to solve the six unknowns. One of these determines the complex viscosity.

The results are derived numerically and presented graphically. From the present work it is possible to deduce results for other models [4-8] as far as they overlap.

Some striking results can be interpreted by considering the influence of the parameters and the frequency on the mechanics of an oscillating straining process.

\section{Formal calculation of the complex viscosity}

\subsection{Volume averaged stress tensor}

For the calculation of the complex viscosity $\eta^{*}$ we will use the general definition:

$$
\langle\bar{T}\rangle \equiv 2 \eta^{*}\langle\boldsymbol{D}\rangle \text {. }
$$

In (1) the deviatoric part $\overline{\langle\boldsymbol{T}\rangle}$ of the volume-averaged stress $\langle\boldsymbol{T}\rangle$ may be evaluated by surface integrals over the particles [11]

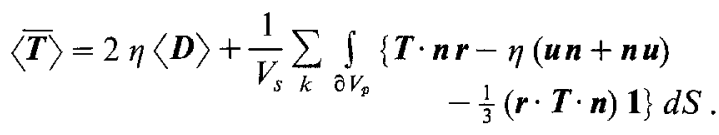

The volume averaged rate of strain tensor $\langle\boldsymbol{D}\rangle$ may be related to the macroscopic velocity at the sample boundaries

$$
\langle\boldsymbol{D}\rangle=\frac{1}{2 V_{\mathrm{s}}} \int_{\partial V_{s}}\left(\boldsymbol{u}^{0} \boldsymbol{n}+\boldsymbol{n} \boldsymbol{u}^{0}\right) d S .
$$

In principle this method gives a complete constitutive equation, but for spherical particles $\eta^{*}$ turns out to be just a scalar function [12].

\subsection{Statistical cell model}

To account for the hydrodynamic interaction between deformable spheres a statistical cell model will be used. In this model it is assumed that the averaged hydrodynamic effect of neighbouring particles may be expressed in boundary conditions that apply at a cer- tain distance $b$ from each particle. This distance can be regarded as the hydrodynamic interaction radius. The concentration dependence of properties calculated with this model is governed by an assumed relation between $b$ and the concentration. This is a semi-empirical relation.

In the low-concentration region a relatively simple expression for $b$ was proposed by Simha [13]:

$$
b=f \cdot a \cdot \Phi^{-1 / 3} .
$$

Thomas [14] compared the results of Simha's calculations with high-shear steady-state viscosity data on suspensions of rigid spheres. He found a good agreement with experimental data if $f=1.111$ for $\Phi<0.15$. Although the statistical cell model is not selfconsistent theoretically [15] it is very useful for describing the concentration dependence.

At $r=b$ we shall use Simha's boundary conditions [12], i.e. the velocity at $r=b$ is equal to the velocity of a homogeneous sample at that place. We only consider the pure straining part of the flow field and neglect the rotational part of it since the particles are assumed to remain almost spherical in the flow. For convenience we will take the harmonically oscillating flow at the boundaries of the sample and cell to be identical with the flow field used by Oldroyd $[4,16]$. Complex notation will be used.

$\boldsymbol{u}^{0}=G\left[\begin{array}{rrr}-1 & 0 & 0 \\ 0 & -1 & 0 \\ 0 & 0 & 2\end{array}\right] \cdot \boldsymbol{r} \exp (\mathrm{i} \omega t)$ for $\left\{\begin{array}{l}\boldsymbol{r} \text { at } \partial V_{c}, \\ \boldsymbol{r} \text { at } \partial V_{s}\end{array}\right.$

\subsection{Hydrodynamics}

Both internal and external fluid are treated as incompressible Newtonian fluids:

$$
\begin{aligned}
& \boldsymbol{\nabla} \cdot \boldsymbol{u}=0, \\
& \boldsymbol{T}=-p \mathbf{1}+2 \eta \boldsymbol{D} .
\end{aligned}
$$

The Reynolds number is taken to be sufficiently small for the creeping-motion equation to be valid.

$$
\eta \nabla^{2} u=\nabla p
$$

Lamb [17] has given the solution of $(6-8)$. The formulae with the symmetry of (5) are most conveniently expressed in spherical coordinates [4]

$$
\begin{gathered}
u_{r}=\left\{\frac{1}{7} A r^{3}+\frac{1}{2} B r^{-2}+2 C r-3 D r^{-4}\right\} \\
\cdot\left\{\frac{3}{2} \cos ^{2} \phi-\frac{1}{2}\right\}\{\exp (i \omega t)\},
\end{gathered}
$$




$$
\begin{array}{r}
u_{\phi}=\left\{\frac{5}{42} A r^{3}+C r+D r^{-4}\right\} \begin{array}{r}
\{-3 \cos \phi \sin \phi\} \\
\cdot\{\exp (\mathrm{i} \omega t)\}, \\
p=\eta\left\{A r^{2}+B r^{-3}\right\}\left\{\frac{3}{2} \cos ^{2} \phi-\frac{1}{2}\right\} \\
\cdot\{\exp (\mathrm{i} \omega t)\}+p_{0} .
\end{array}
\end{array}
$$

The components of the stress tensor that are relevant to the calculation of $\eta^{*}$ are given by

$$
\begin{aligned}
T_{r r}=\eta & \left\{-\frac{1}{7} A r^{2}-3 B r^{-3}+4 C+24 D r^{-5}\right\} \\
& \cdot\left\{\frac{3}{2} \cos ^{2} \phi-\frac{1}{2}\right\}\{\exp (i \omega t)\}-p_{0}, \\
T_{r \phi}=\eta & \left\{\frac{8}{21} A r^{2}+\frac{1}{2} B r^{-3}+2 C-8 D r^{-5}\right\} \\
& \cdot\{-3 \cos \phi \sin \phi\}\{\exp (i \omega t)\} .
\end{aligned}
$$

\subsection{Formal solution of $\eta^{*}$}

It is now possible to express $\eta^{*}$ formally in terms of the constant $B^{(e)}$ of the flow field in the external fluid and the applied rate of strain [7]. The flow field and the pressure field are given as a function of 8 unknown parameters. If there are no net forces acting on the particles and inertia effects are neglected, the choice of the origin has no effect on the evaluation of the integral in (1). Thus it is permissible to choose a different origin for each particle. Using eqs. $(9,10,12,13)$ we find

$$
\begin{array}{r}
\langle\boldsymbol{T}\rangle=2 \eta^{(e)}\langle\boldsymbol{D}\rangle-\frac{1}{V_{s}} \sum_{k} \frac{2 \pi \eta^{(e)} B^{(e)}}{3}\left[\begin{array}{rrr}
-1 & 0 & 0 \\
0 & -1 & 0 \\
0 & 0 & 2
\end{array}\right] \\
\cdot \exp (\mathrm{i} \omega t) .
\end{array}
$$

The specific viscosity of the dispersion may now be expressed in $B^{(e)} / G$, using $(1,5 \mathrm{~b}, 14)$

$$
\eta_{\mathrm{spec}}^{*}=\frac{\eta^{*}-\eta^{(e)}}{\eta^{(e)}}=-\frac{1}{4} \Phi a^{-3} \frac{B^{(e)}}{G}
$$

Thus $\eta^{*}$ can be solved by finding the value of $B^{(e)} / G$. This can be done by formulating a set of eight linear homogeneous equations in the parameters $A^{(i)} ; B^{(i)}$, $C^{(i)}, D^{(i)}, A^{(e)}, B^{(e)}, C^{(e)}$ and $D^{(e)}$ from which $B^{(e)} / G$ can be solved. Two equations follow from the continuity of the velocity at the cell boundary, and another two from the finiteness of the velocity at $r=0$.

The continuity of the velocity and the forces at the boundaries of the shell (in principle eight equations) are reduced to four equations (see sections 4.3 and 4.4).
To formulate equations describing the continuity of the forces across the shell it is necessary to look more accurately to the mechanics of transversely rigid axisymmetric shells.

\section{Mechanics of transversely rigid axisymmetric shells}

\subsection{Forces and moments in shells}

For the description of the mechanics of transversely rigid axisymmetric shells we will closely follow the shell theory described by Seide [10].

A shell of constant thickness is defined by the equation of its middle surface and the magnitude of the thickness. A system of orthogonal curvilinear coordinates for points inside the shell is defined by the coordinates $\alpha$ and $\beta$ corresponding to the lines of curvature on the middle surface and the distance $\zeta$ along the normal to the middle surface (see figure 2).

We will first summarize the differential equations of the three-dimensional theory of elasticity for the region between the bounding surfaces of the shell. To obtain equilibrium equations for stresses in the shell, we consider a small element in the shell bounded by two surfaces parallel to the middle surface of the shell, a distance $d \zeta$ apart, and four adjacent surfaces perpendicular to the middle surface with normals along the lines of curvature. As is usual in small deformation theory, we assume that the effects of geometry changes of the element on equilibrium of forces are negligible. The surface forces on the element of the shell are shown in figure 2.

On three perpendicular adjacent surfaces of the element, $d S_{\alpha}, d S_{\beta}$ and $d S_{\zeta}$ the forces $\boldsymbol{F}_{\alpha}^{(\zeta)}, \boldsymbol{F}_{\beta}^{(\zeta)}$ and $\boldsymbol{F}_{\zeta}^{(\zeta)}$ act, e.g.

$$
\boldsymbol{F}_{\alpha}^{(\zeta)}=\left[\left(\sigma_{\alpha}\right)^{(\zeta)} \boldsymbol{e}_{\alpha}+\tau_{\alpha \beta}^{(\zeta)} \boldsymbol{e}_{\beta}+\tau_{\alpha \zeta}^{(\zeta)} \boldsymbol{e}_{\zeta}\right] g_{\beta}^{(\zeta)} d \beta d \zeta .
$$

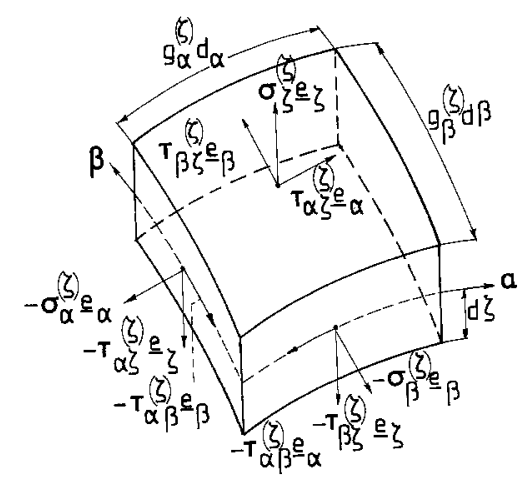

Fig. 2. Surface forces acting on the shell element 
On the three opponent perpendicular surfaces, the forces are given by

$$
\boldsymbol{F}_{\alpha}^{(\zeta)}+\frac{\partial \boldsymbol{F}_{\alpha}^{(\zeta)}}{\partial \alpha} d \alpha, \quad \boldsymbol{F}_{\beta}^{(\zeta)}+\frac{\partial \boldsymbol{F}_{\beta}^{(\zeta)}}{\partial \beta} d \beta, \quad \boldsymbol{F}_{\zeta}^{(\zeta)}+\frac{\partial \boldsymbol{F}_{\zeta}^{(\zeta)}}{\partial \zeta} d \zeta .
$$

When we assume body forces to be absent, we can formulate the vector equation of equilibrium by letting the sum of all the forces on the element be equal to zero. We then obtain

$$
\frac{\partial \boldsymbol{F}_{\alpha}^{(\zeta)}}{\partial \alpha} d \alpha+\frac{\partial \boldsymbol{F}_{\beta}^{(\zeta)}}{\partial \beta} d \beta+\frac{\partial \boldsymbol{F}_{\zeta}^{(\zeta)}}{\partial \zeta} d \zeta=0
$$

This leads to the following equations for the equilibrium of forces

$$
\begin{aligned}
& \frac{\partial}{\partial \alpha}\left[g_{\beta}\left(1+\frac{\zeta}{R_{\beta}}\right) \sigma_{\alpha}^{(\zeta)}\right]+\frac{\partial}{\partial \beta}\left[g_{\alpha}\left(1+\frac{\zeta}{R_{\alpha}}\right) \tau_{\alpha \beta}^{(\zeta)}\right] \\
& +\frac{\partial}{\partial \zeta}\left[g_{\alpha} g_{\beta}\left(1+\frac{\zeta}{R_{\alpha}}\right)\left(1+\frac{\zeta}{R_{\beta}}\right) \tau_{\alpha \zeta}^{(\zeta)}\right] \\
& +\frac{\partial g_{\alpha}}{\partial \beta}\left(1+\frac{\zeta}{R_{\beta}}\right) \tau_{\alpha \beta}^{(\zeta)}-\frac{\partial g_{\beta}}{\partial \alpha}\left(1+\frac{\zeta}{R_{\alpha}}\right) \sigma_{\beta}^{(\zeta)} \\
& +\frac{g_{\alpha} g_{\beta}}{R_{\alpha}}\left(1+\frac{\zeta}{R_{\beta}}\right) \tau_{\alpha \zeta}^{(\zeta)}=0 \\
& \quad \frac{\partial}{\partial \alpha}\left[g_{\beta}\left(1+\frac{\zeta}{R_{\beta}}\right) \tau_{\alpha \beta}^{(\zeta)}\right]+\frac{\partial}{\partial \beta}\left[g_{\alpha}\left(1+\frac{\zeta}{R_{\alpha}}\right) \sigma_{\beta}^{(\zeta)}\right] \\
& +\frac{\partial}{\partial \zeta}\left[g_{\alpha} g_{\beta}\left(1+\frac{\zeta}{R_{\alpha}}\right)\left(1+\frac{\zeta}{R_{\beta}}\right) \tau_{\beta \zeta}^{(\zeta)}\right] \\
& +\frac{\partial g_{\beta}}{\partial \alpha}\left(1+\frac{\zeta}{R_{\alpha}}\right) \tau_{\alpha \beta}^{(\zeta)}-\frac{\partial g_{\alpha}}{\partial \beta}\left(1+\frac{\zeta}{R_{\beta}}\right) \sigma_{\alpha}^{(\zeta)} \\
& +\frac{g_{\alpha} g_{\beta}}{R_{\beta}}\left(1+\frac{\zeta}{R_{\alpha}}\right) \tau_{\beta \zeta}^{(\zeta)}=0, \\
& \frac{\partial}{\partial \alpha}\left[g_{\beta}\left(1+\frac{\zeta}{R_{\beta}}\right) \tau_{\alpha \zeta}^{(\zeta)}\right]+\frac{\partial}{\partial \beta}\left[g_{\alpha}\left(1+\frac{\zeta}{R_{\alpha}}\right) \tau_{\beta \zeta}^{(\zeta)}\right] \\
& +\frac{\partial}{\partial \zeta}\left[g_{\alpha} g_{\beta}\left(1+\frac{\zeta}{R_{\alpha}}\right)\left(1+\frac{\zeta}{R_{\beta}}\right) \sigma_{\zeta}^{(\zeta)}\right] \\
& -\frac{g_{\alpha} g_{\beta}}{R_{\alpha}}\left(1+\frac{\zeta}{R_{\beta}}\right) \sigma_{\alpha}^{(\zeta)}-\frac{g_{\alpha} g_{\beta}}{R_{\beta}}\left(1+\frac{\zeta}{R_{\alpha}}\right) \sigma_{\beta}^{(\zeta)}=0
\end{aligned}
$$

in which the scalar quantities $g_{\lambda}$ and the radii of curvature $R_{\lambda}$ are defined by

$$
\begin{aligned}
& g_{\lambda}=\left(\frac{\partial \boldsymbol{r}}{\partial \lambda} \cdot \frac{\partial \boldsymbol{r}}{\partial \lambda}\right)^{1 / 2} \\
& \frac{1}{R_{\lambda}}=-\frac{1}{g_{\lambda}^{2}} \boldsymbol{e}_{\zeta} \cdot \frac{\partial^{2} \boldsymbol{r}}{\partial \lambda^{2}} .
\end{aligned}
$$

Equilibrium of moments about three orthogonal axes has been satisfied automatically by taking the magnitudes of shear stresses on adjacent faces to be equal i.e.

$$
\begin{aligned}
& \tau_{\alpha \beta}=\tau_{\beta \alpha}, \\
& \tau_{\alpha \zeta}=\tau_{\zeta \alpha}, \\
& \tau_{\beta \zeta}=\tau_{\zeta \beta} .
\end{aligned}
$$

\subsection{Constitutive equations of shells}

The constitutive equations give relations between the stresses and strains in materials. In the linear-elastic shell theory besides these equations usually also straindisplacement relations are given. Assuming the shell thickness to be constant during the deformation, we can describe the strain displacement relations by the following six equations:

$$
\begin{aligned}
\varepsilon_{\alpha}^{(\zeta)}= & \frac{1}{g_{\alpha}\left(1+\frac{\zeta}{R_{\alpha}}\right)}\left[\frac{\partial s_{\alpha}^{(\zeta)}}{\partial \alpha}+\frac{1}{g_{\beta}} \frac{\partial g_{\alpha}}{\partial \beta} s_{\beta}^{(\zeta)}+\frac{g_{\alpha}}{R_{\alpha}} s_{\zeta}^{(\zeta)}\right], \\
\varepsilon_{\beta}^{(\zeta)}= & \frac{1}{g_{\beta}\left(1+\frac{\zeta}{R_{\beta}}\right)}\left[\frac{\partial s_{\beta}^{(\zeta)}}{\partial \beta}+\frac{1}{g_{\alpha}} \frac{\partial g_{\beta}}{\partial \alpha} s_{\alpha}^{(\zeta)}+\frac{g_{\beta}}{R_{\beta}} s_{\zeta}^{(\zeta)}\right], \\
\varepsilon_{\zeta}^{(\zeta)}= & \frac{\partial s_{\zeta}^{(\zeta)}}{\partial \zeta}, \\
\gamma_{\alpha \beta}^{(\zeta)=} & \frac{1}{g_{\alpha}\left(1+\frac{\zeta}{R_{\alpha}}\right)}\left[\frac{\partial s_{\beta}^{(\zeta)}}{\partial \alpha}-\frac{1}{g_{\beta}} \frac{\partial g_{\alpha}}{\partial \beta} s_{\alpha}^{(\zeta)}\right] \\
& +\frac{1}{g_{\beta}\left(1+\frac{\zeta}{R_{\beta}}\right)}\left[\frac{\partial s_{\alpha}^{(\zeta)}}{\partial \beta}-\frac{1}{g_{\alpha}} \frac{\partial g_{\beta}}{\partial \alpha} s_{\beta}^{(\zeta)}\right] \\
\gamma_{\alpha \zeta}^{(\zeta)}= & \frac{\partial s_{\alpha}^{(\zeta)}}{\partial \zeta}+\frac{1}{g_{\alpha}\left(1+\frac{\zeta}{R_{\alpha}}\right)}\left[\frac{\partial s_{\zeta}^{(\zeta)}}{\partial \alpha}-\frac{g_{\alpha}}{R_{\alpha}} s_{\alpha}^{(\zeta)}\right], \\
\gamma_{\beta \zeta}^{(\zeta)=}= & \frac{\partial s_{\beta}^{(\zeta)}}{\partial \zeta}+\frac{1}{g_{\beta}\left(1+\frac{\zeta}{R_{\beta}}\right)}\left[\frac{\partial s_{\zeta}^{(\zeta)}}{\partial \beta}-\frac{g_{\beta}}{R_{\beta}} s_{\beta}^{(\zeta)}\right],
\end{aligned}
$$

in which the displacement vector $s$ is given by

$$
\boldsymbol{s}=s_{\alpha} \boldsymbol{e}_{\alpha}+s_{\beta} \boldsymbol{e}_{\beta}+s_{\zeta} \boldsymbol{e}_{\zeta}
$$

Since the shell material is assumed to be linear-elastic and isotropic in layers parallel to the middle surface, 
we can write the following stress-strain relations.

$$
\begin{aligned}
& \varepsilon_{\alpha}^{(\zeta)}=\frac{1}{E}\left[\sigma_{\alpha}^{(\zeta)}-v \sigma_{\beta}^{(\zeta)}\right]-\frac{\nu_{\zeta}}{E_{\zeta}} \sigma_{\zeta}^{(\zeta)}, \\
& \varepsilon_{\beta}^{(\zeta)}=\frac{1}{E}\left[\sigma_{\beta}^{(\zeta)}-v \sigma_{\alpha}^{(\zeta)}\right]-\frac{v_{\zeta}}{E_{\zeta}} \sigma_{\zeta}^{(\zeta)}, \\
& \varepsilon_{\zeta}^{(\zeta)}=\frac{1}{E_{\zeta}}\left\{\sigma_{\zeta}^{(\zeta)}-v_{\zeta}\left[\sigma_{\alpha}^{(\zeta)}+\sigma_{\beta}^{(\zeta)}\right]\right\}, \\
& \gamma_{\alpha \beta}^{(\zeta)}=\frac{2(1+v)}{E} \tau_{\alpha \beta}^{(\zeta)}, \\
& \gamma_{\alpha \zeta}^{(\zeta)}=\frac{2\left(1+v_{\zeta}\right)}{E_{\zeta}} \tau_{\alpha \zeta}^{(\zeta)}, \\
& \gamma_{\beta \zeta}^{(\zeta)}=\frac{2\left(1+v_{\zeta}\right)}{E_{\zeta}} \tau_{\beta \zeta}^{(\zeta)} .
\end{aligned}
$$

If $E=E_{\zeta}$ and $\nu=v_{\zeta}$ we deal with the conventional Young's modulus and Poisson ratio, respectively.

\subsection{Assumptions for transversely rigid shells}

In most cases, solutions of the equations of threedimensional elasticity theory are extremely difficult to obtain so that approximations to the theory are desirable. The so-called theory of transversely rigid shells is an approximation which replaces the three-dimensional problem by a two-dimensional problem which is somewhat easier to handle. The assumptions underlying this theory are as follows:

The distributions of stresses and displacements in a shell composed of a hypothetical material which has infinite resistance to extension in the direction normal to the middle surface and infinite resistance to shearing in planes normal to the middle surface are an adequate representation of the corresponding distributions in a shell composed of an isotropic material.

These assumptions for transversely rigid shells lead to

$$
\begin{aligned}
& v_{\zeta}=\text { finite }, \\
& E_{\zeta}=\infty .
\end{aligned}
$$

This leads to a reduction of the stress-strain relations $(32-37)$ :

$$
\begin{aligned}
& \varepsilon_{\alpha}^{(\zeta)}=\frac{1}{E}\left[\sigma_{\alpha}^{(\zeta)}-v \sigma_{\beta}^{(\zeta)}\right], \\
& \varepsilon_{\beta}^{(\zeta)}=\frac{1}{E}\left[\sigma_{\beta}^{(\zeta)}-v \sigma_{\alpha}^{(\zeta)}\right], \\
& \gamma_{\alpha \beta}^{(\zeta)}=\frac{2(1+v)}{E} \tau_{\alpha \beta}^{(\zeta)}, \\
& \varepsilon_{\zeta}^{(\zeta)}=\gamma_{\alpha \zeta}^{(\zeta)}=\gamma_{\beta \zeta}^{(\zeta)}=0 .
\end{aligned}
$$

These stress-strain relations correspond to those implied by the assumptions for the deformation attributed to Love and Kirchhoff [18] which can be described as follows:

1. Straight lines normal to the undeformed middle surface remain straight and normal to the deformed middle surface and do not change in length.

2. The normal stresses acting on surfaces parallel to the middle surface may be neglected in comparison with the other stresses.

From these deformation assumptions, it can be derived that the tangential displacements are linear across the shell and that the normal displacements are constant. As a consequence of these assumptions the strain-displacement relations reduce to the following equations expressed in the deformation of the middle surface:

$$
\begin{gathered}
\varepsilon_{\alpha}^{(\zeta)=} \frac{1}{g_{\alpha}\left(1+\frac{\zeta}{R_{\alpha}}\right)}\left[\frac{\partial s_{\alpha}}{\partial \alpha}+\frac{1}{g_{\beta}} \frac{\partial g_{\alpha}}{\partial \beta} s_{\beta}+\frac{g_{\alpha}}{R_{\alpha}} s_{\zeta}\right. \\
\left.-\zeta\left[\frac{\partial \theta_{\alpha}}{\partial \alpha}+\frac{1}{g_{\beta}} \frac{\partial g_{\alpha}}{\partial \beta} \theta_{\beta}\right]\right], \\
\varepsilon_{\beta}^{(\zeta)=} \frac{1}{g_{\beta}\left(1+\frac{\zeta}{R_{\beta}}\right)}\left[\frac{\partial s_{\beta}}{\partial \beta}+\frac{1}{g_{\alpha}} \frac{\partial g_{\beta}}{\partial \alpha} s_{\alpha}+\frac{g_{\beta}}{R_{\beta}} s_{\zeta}\right. \\
\left.-\zeta\left[\frac{\partial \theta_{\beta}}{\partial \beta}+\frac{1}{g_{\alpha}} \frac{\partial g_{\beta}}{\partial \alpha} \theta_{\alpha}\right]\right], \\
\gamma_{\alpha \beta}^{(\zeta)=} \frac{1}{g_{\alpha}\left(1+\frac{\zeta}{R_{\alpha}}\right)}\left[\frac{\partial s_{\beta}}{\partial \alpha}-\frac{1}{g_{\beta}} \frac{\partial g_{\alpha}}{\partial \beta} s_{\alpha}\right. \\
\left.-\zeta\left[\frac{\partial \theta_{\beta}}{\partial \alpha}-\frac{1}{g_{\beta}} \frac{\partial g_{\alpha}}{\partial \beta} \theta_{\alpha}\right]\right] \\
+\frac{1}{g_{\beta}\left(1+\frac{\zeta}{R_{\beta}}\right)}\left[\frac{\partial s_{\alpha}}{\partial \alpha}-\frac{1}{g_{\alpha}} \frac{\partial g_{\beta}}{\partial \alpha} s_{\beta}\right. \\
\left.-\zeta\left[\frac{\partial \theta_{\alpha}}{\partial \beta}-\frac{1}{g_{\alpha}} \frac{\partial g_{\beta}}{\partial \alpha} \theta_{\beta}\right]\right], \\
\varepsilon_{\zeta}^{(\zeta)=} \gamma_{\alpha \zeta}^{(\zeta)}=\gamma_{\beta \zeta}^{(\zeta)}=0,
\end{gathered}
$$

in which

$$
\begin{aligned}
& \theta_{\alpha}=\frac{1}{g_{\alpha}}\left[\frac{\partial s_{\zeta}}{\partial \alpha}-\frac{g_{\alpha}}{R_{\alpha}} s_{\alpha}\right], \\
& \theta_{\beta}=\frac{1}{g_{\beta}}\left[\frac{\partial s_{\zeta}}{\partial \beta}-\frac{g_{\beta}}{R_{\beta}} s_{\beta}\right] .
\end{aligned}
$$

\subsection{Stress resultants and equilibrium conditions}

In a straightforward approach we could express the stresses $\sigma_{\alpha}^{(\zeta)}, \sigma_{\beta}^{(\zeta)}$ and $\tau_{\alpha \beta}^{(\zeta)}$ in terms of the middle surface 
displacements by means of eqs. $(40-42,44-46)$. The equilibrium conditions $(2-4)$ together with the known values of $\sigma_{\zeta}^{(\zeta)}, \tau_{\alpha \zeta}^{(\zeta)}$ and $\tau_{\beta \zeta}^{(\zeta)}$ on the inner and outer parallel bounding surfaces defined by $\zeta= \pm h / 2$ make it possible to solve $\sigma_{\xi}^{(\zeta)}, \tau_{\alpha \zeta}^{(\zeta)}$ and $\tau_{\beta \zeta}^{(\zeta)}$ in terms of the middle surface displacements.

However, it is possible to avoid the consideration of the detailed distributions of stresses in the shell and only deal with the known values of these stresses on the surfaces bounding the shell by integrating the equations of equilibrium over the wall thickness. Stress resultants, expressed as moments and forces per unit length of middle surface, act on the sides of a shell element bounded by the parallel surfaces $\zeta= \pm h / 2$ and planes normal to the middle surface. The sides of this element will be taken in the directions of adjacent lines of curvature. The shell element in figure 2 is only a small portion of this overall shell element.

The force components per unit length of the middle surface can be defined by integrating the appropriate stresses over the cross section on which they act and dividing them by the differential length of the surface line of curvature, see figure 3 . Bending and twisting moment per unit middle surface length are defined similarly, see figure 4 .

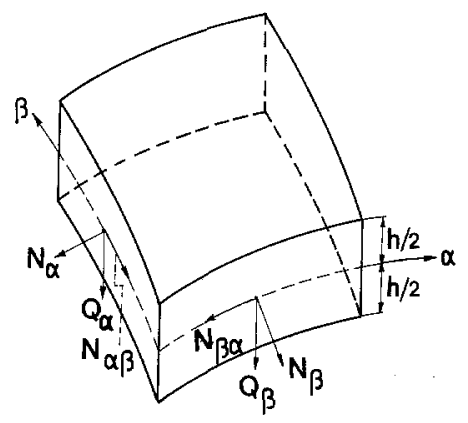

Fig. 3. Resultant force components acting on the shell element

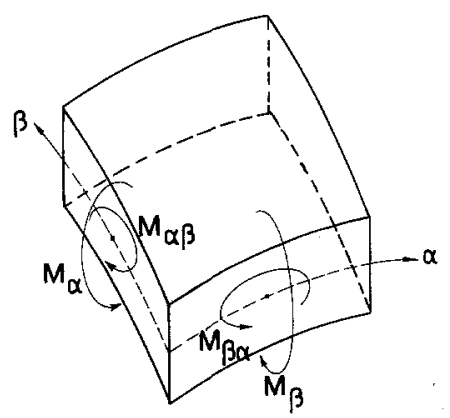

Fig. 4. Resultant moment components acting on the shell element
By integrating the equilibrium conditions $(2-4)$ over the shell thickness we find:

$$
\begin{aligned}
& \frac{\partial}{\partial \alpha}\left(g_{\beta} N_{\alpha}\right)+\frac{\partial}{\partial \beta}\left(g_{\alpha} N_{\beta \alpha}\right)+\frac{\partial g_{\alpha}}{\partial \beta} N_{\alpha \beta}-\frac{\partial g_{\beta}}{\partial \alpha} N_{\beta} \\
& \quad+g_{\alpha} g_{\beta}\left(\frac{Q_{\alpha}}{R_{\alpha}}+q_{\alpha}\right)=0 \\
& \frac{\partial}{\partial \beta}\left(g_{\alpha} N_{\beta}\right)+\frac{\partial}{\partial \alpha}\left(g_{\beta} N_{\alpha \beta}\right)+\frac{\partial g_{\beta}}{\partial \alpha} N_{\beta \alpha}-\frac{\partial g_{\alpha}}{\partial \beta} N_{\alpha} \\
& \quad+g_{\alpha} g_{\beta}\left(\frac{Q_{\beta}}{R_{\beta}}+q_{\beta}\right)=0 \\
& \frac{\partial}{\partial \alpha}\left(g_{\beta} Q_{\alpha}\right)+\frac{\partial}{\partial \beta}\left(g_{\alpha} Q_{\beta}\right)-g_{\alpha} g_{\beta}\left(\frac{N_{\alpha}}{R_{\alpha}}+\frac{N_{\beta}}{R_{\beta}}-q_{\zeta}\right)=0
\end{aligned}
$$

where $q_{\alpha}, q_{\beta}$ and $q_{\zeta}$ are the resultants of surface forces per unit middle surface area acting on the shell element, see figure 5 .

The equilibrium equations for the resulting moments can be obtained in a similar way:

$$
\begin{aligned}
& \frac{\partial}{\partial \alpha}\left(g_{\beta} M_{\alpha}\right)+\frac{\partial}{\partial \beta}\left(g_{\alpha} M_{\beta \alpha}\right)+\frac{\partial g_{\alpha}}{\partial \beta} M_{\alpha \beta}-\frac{\partial g_{\beta}}{\partial \alpha} M_{\beta} \\
& \quad-g_{\alpha} g_{\beta}\left(Q_{\alpha}-m_{\alpha}\right)=0 \\
& \frac{\partial}{\partial \beta}\left(g_{\alpha} M_{\beta}\right)+\frac{\partial}{\partial \alpha}\left(g_{\beta} M_{\alpha \beta}\right)+\frac{\partial g_{\beta}}{\partial \alpha}-M_{\beta \alpha}-\frac{\partial g_{\alpha}}{\partial \beta} M_{\alpha} \\
& \quad-g_{\alpha} g_{\beta}\left(Q_{\beta}-m_{\beta}\right)=0 \\
& N_{\alpha \beta}-N_{\beta \alpha}-\frac{M_{\beta \alpha}}{R_{\beta}}+\frac{M_{\alpha \beta}}{R_{\alpha}}=0
\end{aligned}
$$

where $m_{\alpha}$ and $m_{\beta}$ are the resultant moments of surface forces per unit middle surface area, see figure 6 .

\subsection{Results for axisymmetric shells}

The theory of transversely rigid shells will be used now for the special case of small axisymmetric deformations of spherical shells, and describe the sphere in spherical coordinates $r, \phi, \theta$. The radius of the

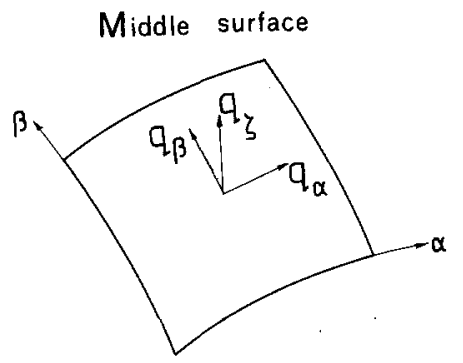

Fig. 5. Resultant external loading force components 


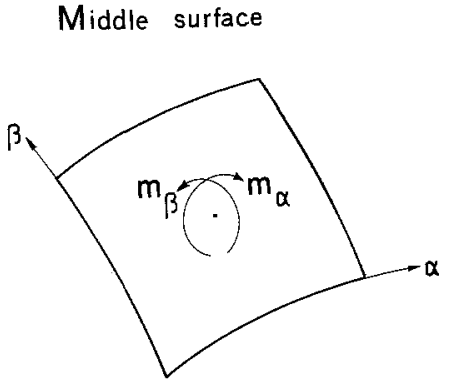

Fig. 6. Resultant external loading moment components

middle surface of the shell is $a_{m}$. We choose the symmetry-axis to be in the $\phi=0$ direction. Thus the following forces are zero

$$
\tau_{\theta r}^{(\zeta)}=\tau_{\phi \theta}^{(\zeta)}=0 .
$$

This leads to:

$$
N_{\theta \phi}=N_{\phi \theta}=Q_{\theta}=M_{\phi \theta}=M_{\theta \phi}=q_{\theta}=m_{\theta}=0 .
$$

The chosen geometry leads to

$$
\begin{aligned}
& R_{\phi}=R_{\theta}=g_{\phi}=a_{m}, \\
& g_{\theta}=a_{m} \sin \phi .
\end{aligned}
$$

The equilibrium equations $(50-55)$ then become for the axisymmetric case:

$$
\begin{aligned}
& \frac{\partial}{\partial \phi}\left(N_{\phi} \sin \phi\right)-N_{\theta} \cos \phi+\left(Q_{\phi}+q_{\phi} a_{m}\right) \sin \phi=0, \\
& \frac{\partial}{\partial \phi}\left(Q_{\phi} \sin \phi\right)-\left(N_{\phi}+N_{\theta}-q_{r} a_{m}\right) \sin \phi=0, \\
& \frac{\partial}{\partial \phi}\left(M_{\phi} \sin \phi\right)-M_{\theta} \cos \phi-\left(Q_{\phi}-m_{\phi}\right) a_{m} \sin \phi=0,
\end{aligned}
$$

where $q_{\phi}, q_{r}$ and $m_{\phi}$ are reduced to:

$$
\begin{aligned}
& q_{\phi}=\left(1+\frac{h}{2 a_{m}}\right)^{2} T_{\phi r}^{(h / 2)}-\left(1-\frac{h}{2 a_{m}}\right)^{2} T_{\phi r}^{(-h / 2)}, \\
& q_{r}=\left(1+\frac{h}{2 a_{m}}\right)^{2} T_{r r}^{(h / 2)}-\left(1-\frac{h}{2 a_{m}}\right)^{2} T_{r r}^{(-h / 2)}, \\
& m_{\phi}=\frac{h}{2}\left(1+\frac{h}{2 a_{m}}\right)^{2} T_{\phi r}^{(h / 2)}+\frac{h}{2}\left(1-\frac{h}{2 a_{m}}\right)^{2} T_{\phi r}^{(-h / 2)} .
\end{aligned}
$$

The stress-strain relations can be described as

$$
\begin{gathered}
N_{\phi}=\frac{E h}{1-v^{2}}\left(\varepsilon_{\phi}+v \varepsilon_{\theta}\right), \\
N_{\theta}=\frac{E h}{1-v^{2}}\left(\varepsilon_{\theta}+v \varepsilon_{\phi}\right),
\end{gathered}
$$

$$
\begin{aligned}
& M_{\phi}=\frac{E h^{3}}{12\left(1-v^{2}\right)}\left(K_{\phi}+v K_{\theta}\right) . \\
& M_{\theta}=\frac{E h^{3}}{12\left(1-v^{2}\right)}\left(K_{\theta}+v K_{\phi}\right) .
\end{aligned}
$$

The strain-deformation relations in this configuration are

$$
\begin{aligned}
\varepsilon_{\phi} & =\frac{1}{a_{m}}\left(\frac{d s_{\phi}}{d \phi}+s_{r}\right), \\
\varepsilon_{\theta} & =\frac{1}{a_{m}}\left(s_{\phi} \cot \phi+s_{r}\right), \\
K_{\phi} & =-\frac{1}{a_{m}^{2}}\left(\frac{d^{2} s_{r}}{d \phi^{2}}-\frac{d s_{\phi}}{d \phi}\right), \\
K_{\theta} & =-\frac{1}{a_{m}^{2}}\left(\frac{d s_{\mathrm{r}}}{d \phi}-s_{\phi}\right) \cot \phi .
\end{aligned}
$$

The eqs. $(60-73)$ make it possible to express the external loading force components $q_{r}$ and $q_{\phi}$ and the external loading moment $m_{\phi}$ in terms of the displacements of the middle surface.

These relations will be used in section 4.4 to derive the equations for the continuity of the forces across the shell.

\section{Boundary conditions for harmonically oscillating pure straining flow}

\subsection{Velocity cell boundary conditions}

As was stated before in section 2.4, we have to formulate a set of eight linear homogeneous equations in the parameters $A^{(i)}, B^{(i)}, C^{(i)}, D^{(i)}, A^{(e)}, B^{(e)}, C^{(e)}$ and $D^{(e)}$ to solve the specific viscosity. The first equations can be derived from the continuity of the velocity at the cell boundary. Outside the cell volume the flow field is taken to be the harmonically oscillating flow used by Oldroyd $[4,16]$. This flow field is described by eq. (5). Inside the cell volume the flow field can be described by eqs. (9) and (10), using the parameters of external flow fields. Continuity of the velocity at the cell boundary leads to

$$
\begin{aligned}
& \frac{1}{7} b^{2} A^{(e)}+\frac{1}{2} b^{-3} B^{(e)}+2 C^{(e)}-3 b^{-5} D^{(e)}=2 G, \\
& \frac{5}{14} b^{2} A^{(e)}+3 C^{(e)}+3 b^{-5} D^{(e)}=3 G .
\end{aligned}
$$




\subsection{Finite velocity conditions}

The next two equations are simply derived from the requirement of finite velocity at $r=0$ :

$$
\begin{aligned}
& B^{(i)}=0, \\
& D^{(i)}=0 .
\end{aligned}
$$

\subsection{Continuity of the velocity across the shell}

We can derive two equations from the continuity condition for the velocity across the shell. The velocity is given by eqs. (9) and (10) using the internal parameters for the inner region, $A^{(i)}, B^{(i)}, C^{(i)}$ and $D^{(i)}$ and using the external parameters $A^{(e)}, B^{(e)}, C^{(e)}$ and $D^{(e)}$ for the velocity in the outer region. These velocities can be related by relations from the shell theory.

Seide [10] gives also a relation between the velocity of the middle surface of the shell and the velocity of a point in the shell wall at a distance $\zeta$ from the middle surface. These relations can be written as follows:

$$
\begin{aligned}
& u_{r}^{(m)}=u_{r}^{(\zeta)}, \\
& u_{\phi}^{(m)}=\frac{R_{\phi}}{R_{\phi}+\zeta} u_{\phi}^{(\zeta)}+\frac{\zeta}{\zeta+R_{\phi}} \frac{\partial u_{r}^{(\zeta)}}{\partial \phi} .
\end{aligned}
$$

Assuming the no-slip condition to be valid the velocities $u_{\phi}^{( \pm h / 2)}, u_{r}^{( \pm h / 2)}$ at the boundary surfaces of the shell are known in terms of $A^{(i)}, \ldots$ and $A^{(e)}, \ldots$ Using eqs. (78) and (79) at $\zeta= \pm h / 2$ we can not only obtain the velocities at the middle surface in terms of the flow field of the inner region, but also in terms of the flow field of the outer region. By the requirement of the continuity of the velocity at the middle surface we thus can obtain two relations:

$$
\begin{gathered}
\frac{1}{7} A^{(i)}\left(a_{m}-\frac{h}{2}\right)^{3}+\frac{1}{2} B^{(i)}\left(a_{m}-\frac{h}{2}\right)^{-2} \\
+2 C^{(i)}\left(a_{m}-\frac{h}{2}\right)-3 D^{(i)}\left(a_{m}-\frac{h}{2}\right)^{-4} \\
=\frac{1}{7} A^{(e)}\left(a_{m}+\frac{h}{2}\right)^{3}+\frac{1}{2} B^{(e)}\left(a_{m}+\frac{h}{2}\right)^{-2} \\
+2 C^{(e)}\left(a_{m}+\frac{h}{2}\right)-3 D^{(e)}\left(a_{m}+\frac{h}{2}\right)^{-4}, \\
\frac{a_{m}}{a_{m}-h / 2}\left\{\frac{5}{42} A^{(i)}\left(a_{m}-\frac{h}{2}\right)^{3}+C^{(i)}\left(a_{m}-\frac{h}{2}\right)\right. \\
\left.+D^{(i)}\left(a_{m}-\frac{h}{2}\right)^{-4}\right\}
\end{gathered}
$$

$$
\begin{gathered}
-\frac{h / 2}{a_{m}-h / 2}\left\{\frac{1}{7} A^{(i)}\left(a_{m}-\frac{h}{2}\right)^{3}+\frac{1}{2} B^{(i)}\left(a_{m}-\frac{h}{2}\right)^{-2}\right. \\
\left.+2 C^{(i)}\left(a_{m}-\frac{h}{2}\right)-3 D^{(i)}\left(a_{m}-\frac{h}{2}\right)^{-4}\right\} \\
=\frac{a_{m}}{a_{m}+h / 2}\left\{\frac{5}{42} A^{(e)}\left(a_{m}+\frac{h}{2}\right)^{3}+C^{(e)}\left(a_{m}+\frac{h}{2}\right)\right. \\
\left.+D^{(e)}\left(a_{m}+\frac{h}{2}\right)^{-4}\right\} \\
+\frac{h / 2}{a_{m}+h / 2}\left\{\frac{1}{7} A^{(e)}\left(a_{m}+\frac{h}{2}\right)^{3}+\frac{1}{2} B^{(e)}\left(a_{m}+\frac{h}{2}\right)^{-2}\right. \\
\left.+2 C^{(e)}\left(a_{m}+\frac{h \cdot}{2}\right)-3 D^{(e)}\left(a_{m}+\frac{h}{2}\right)^{-4}\right\} .
\end{gathered}
$$

\subsection{Equilibrium of the forces in the shell}

In section 3.5 we have written the relations which make it possible to obtain two identities describing the equilibrium of the forces on the shell. Using eqs. (63) and (64) we can write the external loading force components in terms of a jump of components of the stress tensor:

$$
\begin{aligned}
& q_{r}=\left.T_{r r}^{(e)}\right|_{r=a_{m}+h / 2}-\left.T_{r r}^{(i)}\right|_{r=a_{m}-h / 2}, \\
& q_{\phi}=\left.T_{r \phi}^{(e)}\right|_{r=a_{m}+h / 2}-\left.T_{r \phi}^{(i)}\right|_{r=a_{m}-h / 2} .
\end{aligned}
$$

Substituting eqs. (12) and (13) we can write $q_{r}$ and $q_{\phi}$ in terms of the coefficients of the flow field.

Alternative expressions for $q_{r}$ and $q_{\phi}$ in terms of these coefficients can be derived from the eqs. $(60-62)$.

$$
\begin{aligned}
& q_{r}=-\frac{1}{a_{m}}\left\{\frac{\partial Q_{\phi}}{\partial \phi}+\cot \phi Q_{\phi}-N_{\phi}-N_{\theta}\right\}, \\
& q_{\phi}=\frac{1}{a_{m}}\left\{-\frac{\partial N_{\phi}}{\partial \phi}-\cot \phi N_{\phi}+\cot \phi N_{\theta}-Q_{\phi}\right\}
\end{aligned}
$$

in which

$$
Q_{\phi}=\frac{1}{a_{m}}\left\{\frac{\partial M_{\phi}}{\partial \phi}+\cot \phi M_{\phi}-\cot \phi M_{\theta}\right\}+m_{\phi} .
$$

$m_{\phi}$ can be written in terms of the coefficients of the flow field by using eq. (65):

$$
\begin{aligned}
m_{\phi}= & \left.\frac{h}{2}\left(1+\frac{h}{2 a_{m}}\right)^{2} T_{r \phi}^{(e)}\right|_{r=a_{m}+h / 2} \\
& +\left.\frac{h}{2}\left(1-\frac{h}{2 a_{m}}\right)^{2} T_{r \phi}^{(i)}\right|_{r=a_{m}-h / 2}
\end{aligned}
$$

and substituting eqs. (12) and (13). 
The resultant normal forces $N_{\phi}$ and $N_{\theta}$ and the resultant bending moments $M_{\phi}$ and $M_{\theta}$ can be written in terms of the coefficients of the flow field by using eqs. (66-73) and substituting the solutions of the flow field, eqs. (9) and (10).

The expressions for $q_{r}$ and $q_{\phi}$ derived from the forces on the shell caused by the fluid flow have to be equal to the expressions derived from the deformation of the shell. After lengthy calculations this leads to the following identity using $q_{r}$ :

$$
\begin{aligned}
& \frac{1}{2 a_{m}^{2}} \eta^{(e)}\left\{-\frac{1}{7}\left(a_{m}+\frac{h}{2}\right)^{4} A^{(e)}-3\left(a_{m}+\frac{h}{2}\right)^{-1} B^{(e)}\right. \\
& \left.+4\left(a_{m}+\frac{h}{2}\right)^{2} C^{(e)}+24\left(a_{m}+\frac{h}{2}\right)^{-3} D^{(e)}\right\} \\
& -\frac{1}{2 a_{m}^{2}} \eta^{(i)}\left\{-\frac{1}{7}\left(a_{m}-\frac{h}{2}\right)^{4} A^{(i)}-3\left(a_{m}-\frac{h}{2}\right)^{-1} B^{(i)}\right. \\
& \left.+\frac{E}{(\mathrm{i} \omega) 12 h^{3}(5+v)}\left(a_{m}-\frac{h}{2}\right)^{2} C^{(i)}+24\left(a_{m}-\frac{h}{2}\right)^{-3} D^{(i)}\right\} \\
& +\frac{3}{2} a_{m}\left(a_{m}\left(a_{m}-\frac{h}{2}\right)^{-3} a_{m}-\frac{h}{2}\right)^{2} A^{(i)} \\
& \left.+3 a_{m} C^{(i)}-12 a_{m}\left(a_{m}-\frac{h}{2}\right)^{-5} D^{(i)}\right\} \\
& +\frac{E h(1+v)}{(\mathrm{i} \omega) a_{m}^{2}\left(1-v^{2}\right)} \\
& +\frac{3 h}{2 a_{m}^{3}} \eta^{(e)}\left\{\frac{8}{21}\left(a_{m}+\frac{h}{2}\right)^{4} A^{(e)}+\frac{1}{2}\left(a_{m}+\frac{h}{2}\right)^{-1} B^{(e)}\right. \\
& +\frac{\left(3 a_{m}-2 h\right)}{14}\left(a_{m}-\frac{h}{2}\right)^{2} A^{(i)} \\
& \left.+\frac{1}{2}\left(a_{m}+2 h\right)\left(a_{m}+\frac{h}{2}\right)^{2} C^{(e)}-8\left(a_{m}+\frac{h}{2}\right)^{-3} D^{(e)}\right\} \\
& \left.\quad-6\left(a_{m}+\frac{h}{2}\right)\left(a_{m}-\frac{h}{2}\right)^{-3} B^{(i)}-\left(a_{m}-2 h\right) D^{(i)}\right\} \\
& +(i) A^{(i)}+\frac{1}{2}\left(a_{m}-\frac{h}{2}\right)^{-1} B^{(i)}
\end{aligned}
$$

and the identity using $q_{\phi}$ :

$$
\begin{aligned}
& \frac{1}{a_{m}^{2}} \eta^{(e)}\left\{\frac{8}{21}\left(a_{m}+\frac{h}{2}\right)^{4} \cdot A^{(e)}+\frac{1}{2}\left(a_{m}+\frac{h}{2}\right)^{-1} B^{(e)}\right. \\
& \left.+2\left(a_{m}+\frac{h}{2}\right)^{2} C^{(e)}-8\left(a_{m}+\frac{h}{2}\right)^{-3} D^{(e)}\right\} \\
& -\frac{1}{a_{m}^{2}} \eta^{(i)}\left\{\frac{8}{21}\left(a_{m}-\frac{h}{2}\right)^{4} A^{(i)}+\frac{1}{2}\left(a_{m}-\frac{h}{2}\right)^{-1} B^{(i)}\right. \\
& \left.+2\left(a_{m}-\frac{h}{2}\right)^{2} C^{(i)}-8\left(a_{m}-\frac{h}{2}\right)^{-3} D^{(i)}\right\} \\
& =\frac{E h(5+v)}{(\mathrm{i} \omega) a_{m}^{2}\left(1-v^{2}\right)}\left\{\frac{5 a_{m}-3 h}{42}\left(a_{m}-\frac{h}{2}\right)^{2} A^{(i)}\right. \\
& -\frac{h}{4}\left(a_{m}-\frac{h}{2}\right)^{-3} B^{(i)}+\left(a_{m}-h\right) C^{(i)} \\
& \left.+\left(a_{m}+\frac{3}{2} h\right)\left(a_{m}-\frac{h}{2}\right)^{-5} D^{(i)}\right\} \\
& +\frac{E h(1+v)}{(\mathrm{i} \omega) a_{m}^{2}\left(1-v^{2}\right)}\left\{-\frac{1}{7}\left(a_{m}-\frac{h}{2}\right)^{3} A^{(i)}\right. \\
& -\frac{1}{2}\left(a_{m}-\frac{h}{2}\right)^{-2} B^{(i)}-2\left(a_{m}-\frac{h}{2}\right) C^{(i)} \\
& \left.+3\left(a_{m}-\frac{h}{2}\right)^{-4} D^{(i)}\right\} \\
& +\frac{E h^{3}(5+v)}{(i \omega) 12 a_{m}^{4}\left(1-v^{2}\right)}\left\{-\frac{a_{m}}{42}\left(a_{m}-\frac{h}{2}\right)^{2} A^{(i)}\right. \\
& -\frac{1}{2} a_{m}\left(a_{m}-\frac{h}{2}\right)^{-3} B^{(i)} \\
& \left.-a_{m} C^{(i)}+4 a_{m}\left(a_{m}-\frac{h}{2}\right)^{-5} D^{(i)}\right\} \\
& +\frac{h}{2 a_{m}^{3}} \eta^{(i)}\left\{-\frac{8}{21}\left(a_{m}-\frac{h}{2}\right)^{4} A^{(i)}\right. \\
& -\frac{1}{2}\left(a_{m}-\frac{h}{2}\right)^{-1} B^{(i)}-2\left(a_{m}-\frac{h}{2}\right)^{2} C^{(i)} \\
& \left.+8\left(a_{m}-\frac{h}{2}\right)^{-3} D^{(i)}\right\} \\
& +\frac{h}{2 a_{m}^{3}} \eta^{(e)}\left\{-\frac{8}{21}\left(a_{m}+\frac{h}{2}\right)^{4} A^{(e)}\right. \\
& -\frac{1}{2}\left(a_{m}+\frac{h}{2}\right)^{-1} B^{(e)}-2\left(a_{m}+\frac{h}{2}\right)^{2} C^{(e)} \\
& \left.+8\left(a_{m}+\frac{h}{2}\right)^{-3} D^{(e)}\right\} \text {. }
\end{aligned}
$$




\section{Solution and results}

\subsection{Solution of $\eta_{\text {spec }}^{*}$}

As was shown in section 2.4, we can express the specific complex viscosity of the dispersion in terms of the constant $B^{(e)} / G$. This constant is derived from the six boundary conditions derived in section 3 . Therefore we will introduce dimensionless quantities and use a matrix notation. This leads to a notation with six unknowns and five parameters defined as

$$
\begin{aligned}
& Y_{1}=A^{(i)} a_{m}^{2} G^{-1}, \\
& Y_{2}=C^{(i)} G^{-1}, \\
& Y_{3}=A^{(e)} a_{m}^{2} G^{-1}, \\
& Y_{4}=B^{(e)} a_{m}^{-3} G^{-1}, \\
& Y_{5}=C^{(e)} G^{-1}, \\
& Y_{6}=D^{(e)} a_{m}^{-5} G^{-1}, \\
& R=b a_{m}^{-1}, \\
& L=h\left(2 a_{m}\right)^{-1}, \\
& V=\eta^{(i)} \cdot\left(\eta^{(e)}\right)^{-1}, \\
& Z=2 \omega \eta^{(e)} a_{m}(E h)^{-1},
\end{aligned}
$$

and the Poisson ratio $v$.

Instead of the parameter $R$, which is very useful in the description of the mechanics, we can also use the volume fraction $\Phi_{\text {ext }}$ which is more useful in the graphical representation of the results.

$$
\Phi_{\mathrm{ext}}=f^{3}(1+L)^{3} R^{-3} .
$$

Here $\Phi_{\text {ext }}$ is the volume fraction of the particles including the shell.

Substituting these quantities in the six boundary conditions we obtain:

$$
\begin{aligned}
& \frac{1}{7} R^{2} Y_{3}+\frac{1}{2} R^{-3} Y_{4}+2 Y_{5}-3 R^{-5} Y_{6}=2, \\
& \frac{5}{14} R^{2} Y_{3}+3 Y_{5}+3 R^{-5} Y_{6}=3, \\
& \frac{1}{7}(1-L)^{3} Y_{1}+2(1-L) Y_{2}-\frac{1}{7}(1+L)^{3} Y_{3} \\
& -\frac{1}{2}(1+L)^{-2} Y_{4}-2(1+L) Y_{5}+3(1+L)^{-4} Y_{6}=0, \\
& \frac{1}{42}(5-6 L)(1-L)^{2} Y_{1}+(1-2 L) Y_{2} \\
& -\frac{1}{42}(5+6 L)(1+L)^{2} Y_{3}-\frac{1}{2} L(1+L)^{-3} Y_{4} \\
& -(1+2 L) Y_{5}-(1-3 L)(1+L)^{-5} Y_{6}=0,
\end{aligned}
$$

$$
\begin{aligned}
& \left\{\left(-\frac{1}{14}+\frac{8}{7} L\right)(1-L)^{4} V-i \frac{1}{21} \frac{5+v}{1-v^{2}} L^{2}(1-L)^{2} Z^{-1}\right. \\
& \left.+i \frac{1}{7} \frac{1+v}{1-v^{2}}(3-4 L)(1-L)^{2} Z^{-1}\right\} Y_{1} \\
& +\left\{(2+6 L)(1-L)^{2} V-i 2 \frac{5+v}{1-v^{2}} L^{2} Z^{-1}\right. \\
& \left.+i 2 \frac{1+v}{1-v^{2}}(1-4 L) Z^{-1}\right\} Y_{2} \\
& +\left(\frac{1}{14}+\frac{8}{7} L\right)(1+L)^{4} Y_{3}+\frac{3}{2} Y_{4} \\
& +(-2+3 L)(1+L)^{2} Y_{5}-(12+24 L)(1+L)^{-3} Y_{6}=0,(105) \\
& \left\{\frac{8}{21}(1-L)^{5} V+i \frac{1}{63} \frac{5+v}{1-v^{2}}\left(L^{2}+18 L-5\right)\right. \\
& \left.+(1-L)^{2} Z^{-1}+i \frac{2}{7} \frac{1+v}{1-v^{2}}(1-L)^{3} Z^{-1}\right\} Y_{1} \\
& +\left\{2(1-L)^{3} V+i \frac{2}{3} \frac{5+v}{1-v^{2}}\left(L^{2}+6 L-3\right) Z^{-1}\right. \\
& +\frac{1}{2} Y_{4}-2(1+L)^{3} Y_{5}+8(1+L)^{-2} Y_{6}=0 .
\end{aligned}
$$

The specific complex viscosity of the dispersion can be written as (see eq. (15))

$$
\eta_{\mathrm{spec}}^{*}=-\frac{1}{4} f^{3} R^{-3} Y_{4}
$$

$Y_{4}$ can be solved most easily by using the matrix notation of the equations $(101-106)$ :

$$
\boldsymbol{A} \cdot \boldsymbol{Y}=\boldsymbol{C},
$$

where $A$ is a $6 \times 6$ matrix and $Y$ and $C$ are $6 \times 1$ matrices. $Y_{4}$ can be solved by Kramers rule:

$$
Y_{4}=\frac{\operatorname{det}(\boldsymbol{B})}{\operatorname{det}(\boldsymbol{A})},
$$

where the matrix $\boldsymbol{B}$ can be derived from the matrices $\boldsymbol{A}$ and $C$ by substituting $C$ in the fourth column of the matrix $A$. One can solve $Y_{4}$ analytically with the symbolic computer language Reduce II [19, 20].

\subsection{Analytical solution}

The analytical solution of the quantity $Y_{4}$ can be written with the quantity $Z$, a dimensionless angular 
frequency, as the running variable, in the following way

$$
Y_{4}=\frac{X_{1}(Z i)^{2}+X_{2}(Z i)+X_{3}}{X_{4}(Z i)^{2}+X_{5}(Z i)+X_{6}}
$$

where $X_{1}-X_{6}$ are functions of $R, L, V$ and $v$. The analytical solutions of these functions could be given, but are rather lengthy.

The form of this solution is in accordance with the expectations on the basis of the form of general linear viscoelastic equations with some additional considerations. Axelrad [21] demonstrated that assuming the validity of the principle of passivity four categories of linear constitutive equations can be identified. Taking into account that for a dispersion of capsules as considered $\lim _{\omega \rightarrow 0} \omega \eta^{\prime \prime}=0$ and $\lim _{\omega \rightarrow \infty} \eta^{\prime} \neq 0$ only one category remains. The specific viscosity $\eta_{\mathrm{spec}}^{*}$ of (107) with $Y_{4}$ as given in (110) belongs to this category. It is characterized by two retardation and two relaxation times.

In general the analytical solution of $\eta^{*}$ is too cumbersome to be manageable. Only the case $L=0$ gives manageable results. To be able to find the limit for $L \rightarrow 0$ while the interface obtains two-dimensional elastic properties, the specific dynamic viscosity has been expressed in $Z$. Thus it is possible to take $L=0$ in $(101-106)$ while in $Z$ (see eq. (99)) $E h$ is retained as a two-dimensional elasticity. If in the following pages the case $L=0$ is considered, the preceding condition is meant.

To obtain some insight into the general peculiarities of the model $\eta_{\mathrm{spec}}^{*}$ was calculated numerically.

\subsection{Numerical results}

In this section some numerical results of this model will be discussed. These results are given graphically with $Z$ as the running variable and $\eta_{\text {spec }}^{\prime}$ and $\eta_{\text {spec }}^{\prime \prime}$ as the rheological functions considered. They are defined by:

$$
\eta_{\mathrm{spec}}^{*}=\eta_{\mathrm{spec}}^{\prime}-i \eta_{\mathrm{spec}}^{\prime \prime} \text {. }
$$

The correction factor $f$ defined in eq. (4) is given the value 1.111 (see section 2.2). In all examples $v$ is chosen such that the two transitions in $\eta_{\text {spec }}^{*}$ are separated clearly. The maximum value of $L$ is taken to be 0.1 to show clearly the influence of $L$. However one should bear in mind that this shell theory is assumed to be valid only for $L \leqq 0.05$.

An example of the influence of the parameter $V$ is given in figure 7. The most remarkable effect of an increase of $V$ is an increase of the level of the specific viscosity at higher frequencies. Besides it results in a shift of the relaxation times to longer times.

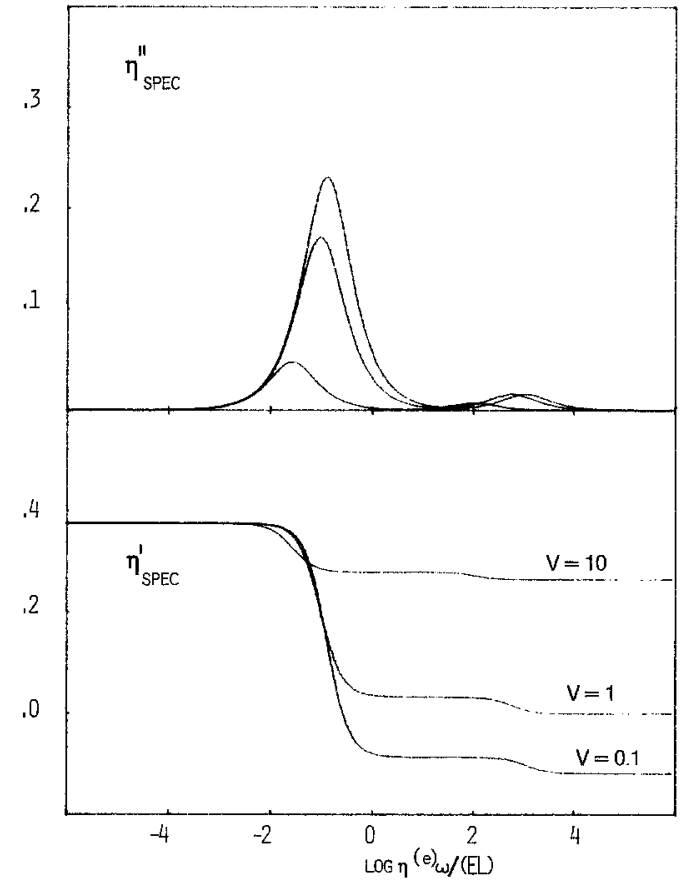

Fig. 7. Influence of the parameter $V$ on the specific viscosity of a dispersion. Parameter values: $\Phi=0.10, L=0, v=0.999$, for $V=10, V=1, V=0.1$

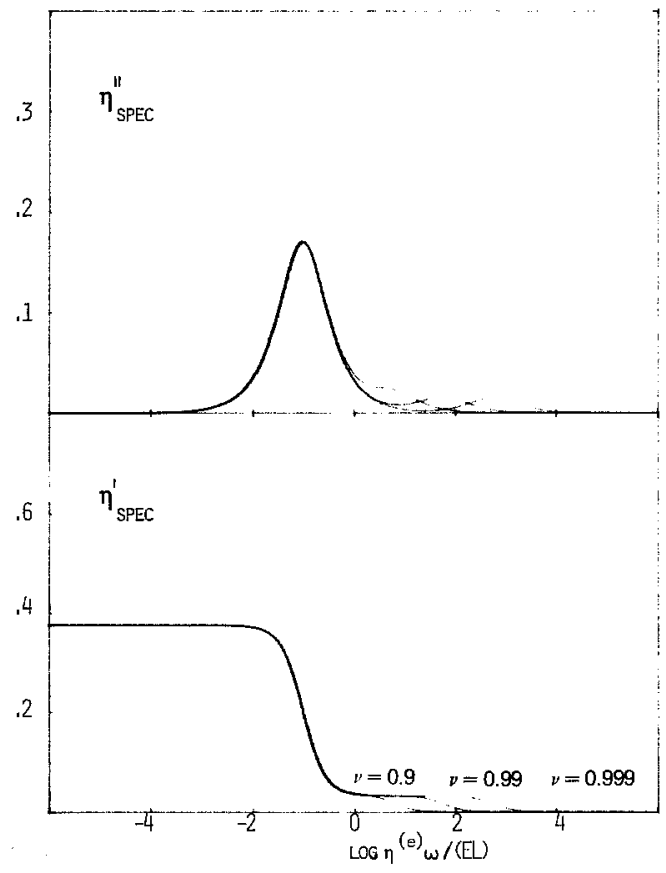

Fig. 8. Influence of the parameter $v$ on the specific viscosity of a dispersion. Parameter values: $\Phi=0.10, L=0, V=1$, for $v=0.999, v=0.99, v=0.9$ 


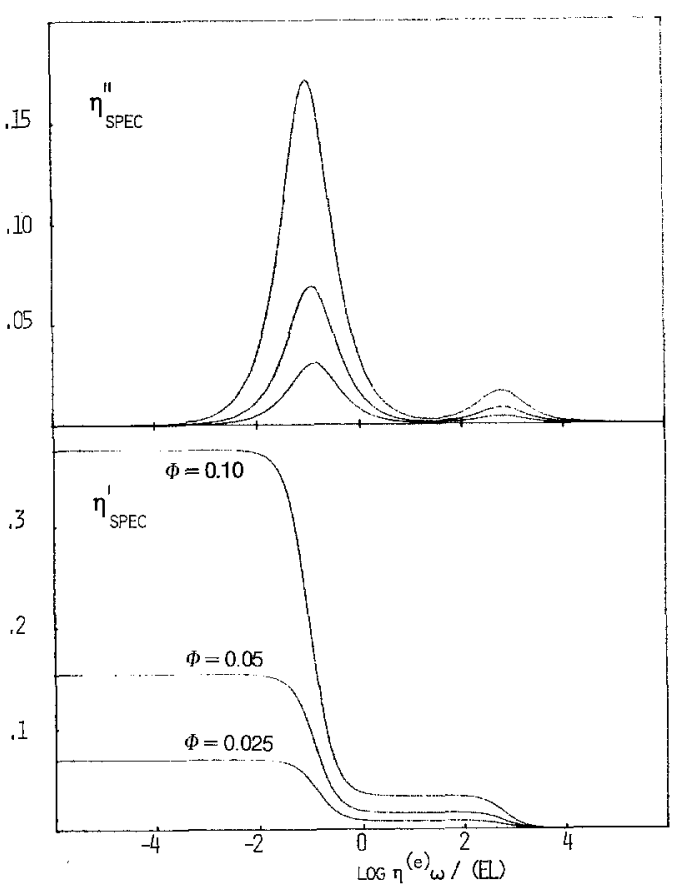

Fig. 9. Influence of the parameter $\Phi$ on the specific viscosity of a dispersion. Parameter values: $L=0, V=1, v=0.999$, for $\Phi=0.10, \Phi=0.05, \Phi=0.025$

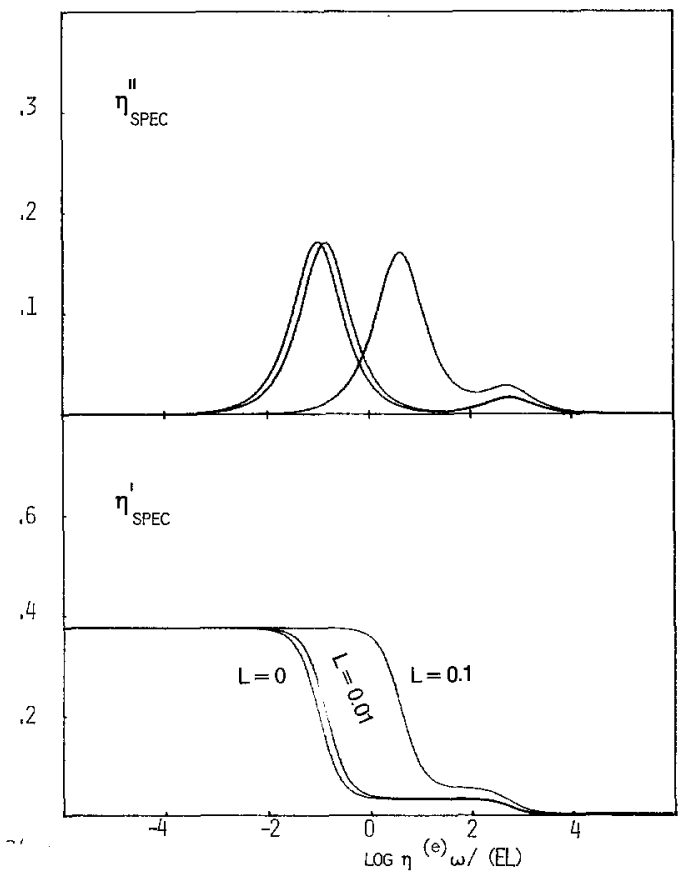

Fig. 10. Influence of the parameter $L$ on the specific viscosity of a dispersion. Parameter values: $\Phi_{\mathrm{ext}}=0.10, V=1, v=0.999$, for $L=0, L=0.01, L=0.1$

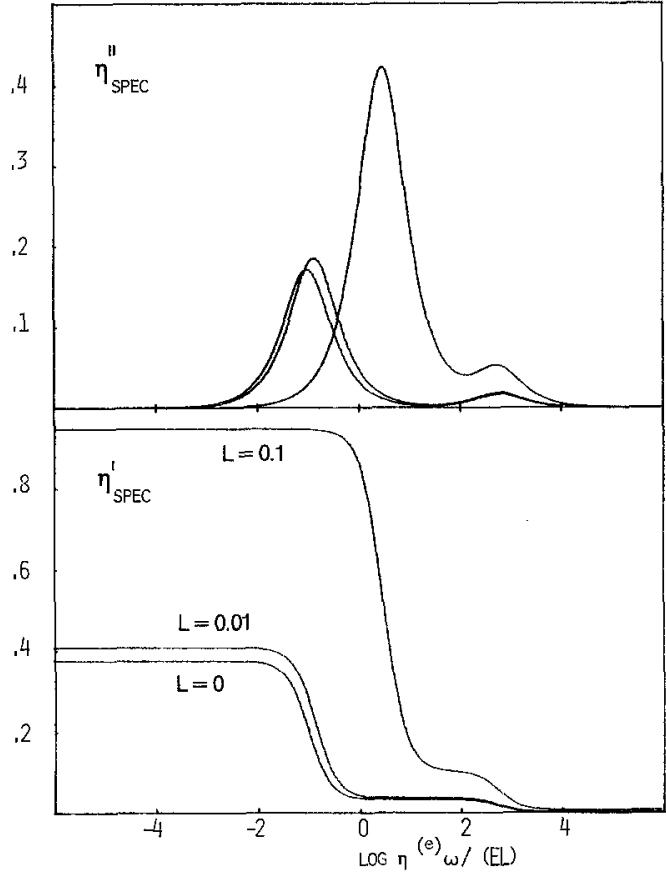

Fig. 11. Influence of the parameter $L$ on the specific viscosity of a dispersion. Parameter values: $\Phi_{\text {int }}=0.1, V=1, v=0.999$, for $L=0, L=0.01, L=0.1$. Here $\Phi_{\text {int }}$ is the volume of the liquid enclosed within the shell

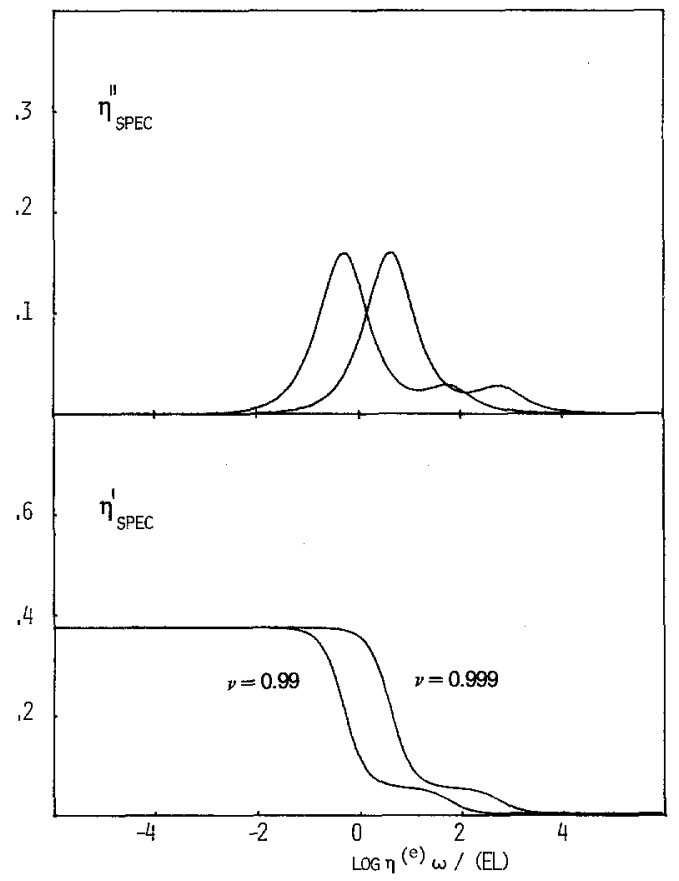

Fig. 12. Influence of the parameter $v$ on the specific viscosity of a dispersion with $L=0.1$. Other parameter values: $\Phi_{\text {ext }}=$ $0.1, V=1$, for $v=0.999$ and $v=0.99$ 
An example of the influence of the parameter $v$ is given in figure 8 . This parameter does not influence the level of the specific viscosity at high or low frequencies. Its influence on the relaxation times is significant. The shortest relaxation time decreases by about one decade if the value $1-v$ decreases by one decade. The longest relaxation time does not change much. Only at higher values of the parameter $L$ does it decrease significantly if the value $1-v$ decreases and for $L=0.1$ both the shortest and the longest relaxation time shift by about the same factor if $1-v$ changes (see figure 12).

The influence of the volume fraction $\Phi$ is visible as a change in magnitude of $\eta^{*}$ over the whole frequency range, see figure 9 . The relaxation times are slightly influenced by the volume fraction. An increase of the volume fraction results in an increase of the relaxation times. The longest relaxation time appears (numerically) to be more sensitive then the shortest one.

The influence of $L$ is the most remarkable result of this model. From the numerical results it appears that an increase of $L$ has a very significant effect on the relaxation times and on the level of the specific viscosity between the two relaxation times. There is also a small increasing effect on the level of the specific viscosity at high frequencies. Examples are given in figure 10,11 and 12 . The longest relaxation time decreases appreciably with increasing shell thickness. The influence on the shortest relaxation time is much smaller (not visible in the given figure but numerically it is significant). The level of the specific viscosity between the two relaxation times increases according to the increase in the shell thickness.

\section{Discussion}

\subsection{Comparison with the literature}

The results obtained can be compared with those from the models given in the literature. The present model is for $L=0$ identical to Oosterbroek's model [7] if in his model the interfacial tension and the interfacial viscosities are taken to be zero. The two-dimensional parameters $\varkappa$ (surface dilatational modulus) and $\mu$ (surface shear modulus) are given by

$$
\varkappa=\frac{E L}{1-v} a_{m}
$$

and

$$
\mu=\frac{E L}{1+v} a_{m} .
$$

The identity is self-evident if one considers the six boundary conditions $(101-106)$ with $L=0$ and the corresponding conditions in [7]. It has been proved [7] that for $\Phi \rightarrow 0$ Oldroyd's model follows from Oosterbroek's model.

A special case of Oldroyd's model should be comparable with the complex viscosity deducible from the linear part of the constitutive equations for a capsule dispersion derived by Barthès-Biesel and Chhim [8]. They studied capsules with an incompressible, homogeneous and isotropic shell of finite thickness characterized by a shear modulus. The validity of their constitutive equation is restricted to a slow varying flow. Thus the deducible complex viscosity is valid for low frequencies. In first order of the frequency, thickness and volume fraction they find

$$
\eta^{\prime}=\eta^{(e)}\left(1+\frac{5}{2} \Phi\right)
$$

and

$$
\eta^{\prime \prime}=\frac{95}{4} \eta^{(e)} \omega \frac{a \eta^{(e)}}{E h} \Phi .
$$

This result can be derived from Oldroyd's model as follows. From the analysis of the present work it appeared that for the transition from three dimensions to two dimensions (112) and (113) must be used. To incorporate the three-dimensional incompressibility, as assumed by Barthès-Biesel and Chhim, obviously $v=\frac{1}{2}$ should be taken instead of $v=1$ for two-dimensional incompressibility. Indeed then (114) and (115) follow from [4].

Comparison with the work of Sakanishi and Takano [5] and Brunn [6] is also possible if explicit formulae have been given. They studied the same kind of capsules as Barthès-Biesel and Chhim did and derived in first order of volume fraction the complex viscosity and the linear constitutive equation for the capsule dispersion, respectively. In general four relaxation times are found. This can be expected because at each shell surface two force balance equations apply. For $L \ll 1$ two relaxation times are expected to be equal to those found by Oldroyd. It appears that the two longest relaxation times can be identified as those occurring for a twodimensional shell. This can be demonstrated by comparing the relaxation times found by Oldroyd, again using $(112,113)$ and $v=\frac{1}{2}$, with those explicitly given in [5] and [6] for $h \rightarrow 0$. The times are given by

$\tau_{l, s}=\frac{3 a \eta_{0}}{5 E h}\left(1 \mp \sqrt{1-\frac{96 \eta_{0}}{25\left(24 \eta^{(e)}+19 \eta^{(i)}\right)}}\right)^{-1}$

with

$\eta_{0}=\frac{\left(3 \eta^{(e)}+2 \eta^{(i)}\right)\left(16 \eta^{(e)}+19 \eta^{(i)}\right)}{\left(24 \eta^{(e)}+19 \eta^{(i)}\right)}$ 
Brunn [6] gave the same times. Sakanishi and Takano [5] gave the limit cases $\eta^{(e)} / \eta^{(i)} \rightarrow 0$ and $\eta^{(e)} / \eta^{(i)} \rightarrow \infty$. The physical origin of the other two times, which are for $L<0.05$ at least a factor 100 shorter, should be due to the three-dimensionality of the membrane.

In a more recent paper Takano and Sakanishi [9] studied capsules with shells characterized by anisotropic elastic properties. For these capsules also two relaxation times are found. They are difficult to compare with the results of the present work not only because numerically derived results are difficult to compare but their relaxation effects (so-called negative relaxations) are not compatible with the principle of passivity [21].

\subsection{Interpretation of the results}

In the following some striking aspects of the presented results are interpreted.

The ratio $V$ influences $\eta^{*}$ in different ways (see figure 7). The relaxation times become longer and the magnitude of the transition decreases if $V$ increases. A relaxation time can be written as a positive constant multiplied by an effective viscosity and divided by an effective elasticity. In this case the effective viscosity is a monotonically increasing function of $\eta^{(i)}$ and of $\eta^{(e)}$ [7]. The effective elasticity is either $E /(1-v)$ or $E /(1+v)$. The first fact causes an increase of the relaxation times with increasing $V$. At sufficiently high frequencies the stresses acting at each side of the shell become much larger than the difference between them due to the elastic deformation as the deformation of the shell cannot become larger than the overall applied strain. As a consequence the high frequency limit of $\eta^{\prime}$ can be described by a model in which the interfacial mechanical properties are negligible (see e.g. [22]). For $V \sim O(1)$ the limit will be almost proportional to the volume fraction. For $V \rightarrow \infty \eta^{\prime}(\omega \rightarrow \infty)$ will become equal to $\eta^{\prime}(\omega \rightarrow 0)$ while the maximum of $\eta^{\prime \prime}$ decreases.

In figure 8 the effect of $v$ is shown. Since for $L=0$ the longest relaxation time $\tau_{l}$ is proportional to $\mu^{-1}$ and the shortest $\tau_{s}$ to $\varkappa^{-1}$ [7] according to eqs. (112) and (113) $\tau_{l}$ changes with $(1+v)$ and $\tau_{s}$ with $(1-v)$.

The influence of the volume fraction is shown in figure 9. The viscosity $\eta^{\prime}(\omega=0)$ equals Simha's steadystate viscosity for solid spheres (see also [7]). The explanation is as follows: For $\omega \rightarrow 0$ the viscous stresses in the forces equilibrium conditions become zero. As a consequence the jump in the viscous stresses due to the elastic deformation of the shell should also vanish. Since $E$ is finite the deformation of the shell becomes zero and the capsule dispersion becomes indistinguishable from a suspension of solid spheres. It is not surprising that this correspondence was also found for finite $L$ within numerical accuracy. Since $\eta^{\prime}(\omega=0)$ increases strongly in a non-linear way with $\Phi$ the magnitude of the transitions should increase strongly.

Finite $L$ has an effect on the relaxation times and the magnitude of $\eta^{*}$ (figures 10,11 and 12). The difference between figure 10 and 11 is mainly seen in the magnitude difference at a fixed frequency. It can be explained largely by the influence of the volume fraction. In figure $10 \Phi_{\text {ext }}$ includes the shell volume while in figure $11 \Phi_{\text {int }}$ is just the volume fraction of the dispersed phase enclosed in the shells. Changing $L$ if $\phi_{\text {int }}$ is constant implies changing $\phi_{\mathrm{ext}}$, so $\eta^{\prime}(\omega \rightarrow 0)$ increases (see the preceding comment on figure 9 ).

The main influence of $L$ itself for a fixed value of $v$ is a large shift of $\tau_{l}$ and a small shift of $\tau_{s}$. This can be understood by consideration of the occurring forces and ensueing deformation. After some calculations starting from eqs. (84) and (85) it follows that the angle-independent elastic part in the force equilibria can be written as:

$$
q_{r}=\varkappa g_{A}(a, L)+(3 \varkappa+2 \mu) L^{2} g_{B}(a, L)
$$

for the $T_{r r}$ jump condition and

$$
\begin{aligned}
q_{\phi}= & x g_{A}(a, L)+\frac{1}{3}(3 x+2 \mu) L^{2} g_{B}(a, L) \\
& +\mu\left(g_{A}(a, L)-g_{B}(a, L)\right)
\end{aligned}
$$

for the $T_{r \phi}$ jump condition.

Here $g_{A}$ and $g_{B}$ are functions which can be deduced from eqs. (88) and (89). As already stated the elastic deformation of the capsule will vanish for $\omega \rightarrow 0$ and hence $g_{A}(\omega \rightarrow 0)=0$ and $g_{B}(\omega \rightarrow 0)=0$. For $L=0$ and $v \sim 1$ it has been demonstrated [7] that $\tau_{l}$ is (mainly) determined by $\mu$. This can be realized if $q_{r}(a, 0) \sim 0$ and $q_{\phi}(a, 0) \sim-\mu g_{B}(a, 0)$ is left. Figure 8 confirms that $\tau_{l}$ is hardly influenced by $\varkappa_{\text {. For finite but small }}$ $L(<0.1)$ one can assume that $g_{A}(a, L) \sim g_{A}(a, 0)$. For $\omega \rightarrow 0$ again $q_{r} \rightarrow 0$. Assuming that also for finite $L$ the condition for $\tau_{l}$ is still such that $q_{r} \sim 0$ then

$$
\begin{aligned}
g_{\phi} \sim & -\frac{2}{3}(3 x+2 \mu) L^{2} g_{B}(a, L) \\
& +\mu\left(g_{A}(a, L)-g_{B}(a, L)\right) .
\end{aligned}
$$

In figures 10 and 11 the maximum value of $L=0.1$ and $v=0.999$. Then $\frac{2}{3}(3 x+2 \mu) L^{2} \sim 2 x L^{2} \sim 40 \mu$. This would imply that $\tau_{l}$ for $L=0.1$ is mainly due to $x L^{2}$ and no longer to $\mu$ if $\left|g_{B}(a, L)\right|$ is of the same order of magnitude as $\left|g_{A}(a, L)\right|$. The shift of $\tau_{l}$ between $L=0$ and $L=0.1$ in figures 10 and 11 is in agreement with this interpretation.

A stronger test for this type of interpretation follows using the fact that as observed $\tau_{s}$ is hardly influenced by $L$. This is plausible by consideration of (118) and 
(119). Assuming that $g_{A}(a, 0) \sim g_{A}(a, L), g_{B}(a, 0)$ $\sim g_{B}(a, L),\left|g_{A}\right| \sim\left|g_{B}\right|, v \sim 1, x \gg \mu$ and $L$ maximal $0.1, q_{r} \sim \varkappa g_{A}(a, L)$ and $q_{\phi} \sim \varkappa g_{A}(a, L)$. Thus on a $\log$ scale no appreciable shift of $\tau_{s}$ due to a change in $L$ is to be expected. Combining the interpretations of $\tau_{l}$ and $\tau_{s}$ for fixed $a, L$ and $\Phi$, and if one expects that if $1-v$ changes by some factor (e.g. 0.01 to 0.001 ) $\tau_{l}$ and $\tau_{s}$ should change by about the same factor in the same direction. This is confirmed numerically in figure 12. It may be concluded that $\tau_{s}$ is almost independent of $L$ and that $\tau_{l}$ decreases strongly as a consequence of resistance against bending in the case of finite thickness. This reasoning is only valid if $v \sim 1$, as otherwise a combination of effects can be expected.

It may be interesting to note that the origin of the shift of $\tau_{l}$ is the influence of the coefficient of flexural rigidity $E h^{3} /\left(12\left(1-v^{2}\right)\right)$ in (88) and (89). Kléman [23] has pointed out that the energy connected with the pertinent deformation is of the same form as the splay energy (energy of curvature) given by De Gennes [24] and Helfrich [25]. Apparently complex viscosity measurements, e.g. on vesicles, might be a tool for the investigation of a curvature effect.

In conclusion it can be said that the present model shows that finite thickness of the shell can strongly influence the relaxation times. The model can be used for the interpretation of the linear viscoelastic behaviour of a dispersion of hydrodynamically interacting capsules at finite volume concentration. Its significance with respect to other available models is explained.

\section{Acknowledgement}

Thanks are due to Mr. R. Tropper for his preliminary studies on this subject.

\section{References}

1. Oosterbroek M, Mellema J, Lopulissa JS (1981) J Colloid Interface Sc 84:27

2. Blom C, Mellema J (1984) J Disp Sc Techn 5:193

3. Bredimas M, Veyssié M (1983) J Non Newtonian Fluid Mech 12:165
4. Oldroyd JG (1955) Proc Roy Soc London Ser A 232:567

5. Sakanishi A, Takano Y (1974) Jap J Appl Phys 13:882

6. Brunn P (1980) Biorheology 17:419

7. Oosterbroek M, Mellema J (1981) J Colloid Interface Sc $84: 14$

8. Barthès-Biesel D, Chhim V (1981) Int J Multiphase Flow 7:493

9. Takano Y, Sakanishi A (1982) Biorheology 19:599

10. Seide P (1975) Small elastic deformations of thin shells. Noordhof int. publishing, Leiden, Holland

11. Landau LD, Lifshitz EM (1959) Fluid Mechanics p 76, Pergamon Press, Oxford

12. Brenner H (1972) Suspension rheology. In: Schowalter (ed) Progress in heat and mass transfer, vol 5. Pergamon Press, Oxford

13. Simha R (1952) J Appl Phys 23:1020

14. Thomas DG (1965) J Colloid Interface Sc 20:267

15. Safrai VM (1970) J Appl Mech Tech Phys 11:188

16. Oldroyd JG (1953) Proc Roy Soc London Ser A 218:122

17. Lamb H (1975) Hydrodynamics, p 596. Cambridge University Press, London

18. Love AEH (1944) A treatise on the mathematical theory of elasticity (4 e.d., chapter 24). Dover Publications, New York

19. Barthès-Biesel D, Acrivos A (1973) J Comput Phys 12:403

20. Hearn AC (1973) Reduce 2 Users manual. University of Utah, Salt Lake City, Utah

21. Axelrad DR (1970) Adv in Molec Relaxation Processes 2:41

22. Mellema J, Willemse MWM (1983) Physica 122A:286

23. Kléman M (1976) Proc Roy Soc London Ser A 347:387

24. De Gennes PG (1969) J Phys 30:65, C-4

25. Helfrich W (1973) Z Naturforschung 28C:693

(Received April 18, 1984)

Authors' addresses:

R. A. de Bruijn

Unilever Research Laboratory

P.O. Box 114

NL-3130 AC Vlaardingen

Dr. J. Mellema*)

Department of Applied Physics

Twente University of Technology

P.O. Box 217

NL-7500 AE Enschede

*) To whom correspondence should be addressed 